\title{
Recurrent Primary Amyloidosis
}

National Cancer Institute

\section{Source}

National Cancer Institute. Recurrent Primary Amyloidosis. NCI Thesaurus. Code C133732.

Reemergence of primary amyloidosis after a period of remission. 